\title{
Einsatz elektronischer Kleinrechner im klinisch-chemischen Zentrallaboratorium
}

\author{
VON K. BORNER \\ Aus dem Institut für Angewandte Pbysiologische Chemie und Kliniscbe Chemie der Freien Universität Berlin \\ (Direktor: Prof. Dr. H.-J. Dulce)
}

(Eingegangen am 11. März 1968)

Herrn Prof. Dr. Dr. Ernst Scbiitte zum 60. Geburtstag gewidmet

\begin{abstract}
Es wird ein preiswertes System zum Verarbeiten von Daten aus dem klinisch-chemischen Laboratorium vorgeschlagen. Für das Berechnen von Ergebnissen wird ein elektronischer Kleinrechner (Olivetti Programma 101 oder 203P) eingesetzt. Geeignete Programmbeispiele, speziell für den Auto-Analyzer, werden zusammen mit Arbeitsvorschriften beschrieben.
\end{abstract}

A price-worthy system is described for processing data from the clinical-chemical laboratory. An electronic bench calculator (Olivetti Programma 101 or 203P) is used. Examples of suitable programs, designed for the autoanalyser are described, together with working instructions.

An das klinisch-chemische Zentrallaboratorium werden ständig steigende Anforderungen in bezug auf die Anzahl der gewünschten Analysen gestellt. Diese Situation erzwingt alle erdenklichen Rationalisierungsmaßnahmen. Durch Mechanisieren (englisch ,automation") des Analysenvorgangs und der Messung, z. B. durch den Einsatz von Technicon-Autoanalyzern, läßt sich meistens eine größere Anzahl von Proben pro Tag als mit manuellen Methoden bearbeiten. Infolge des erhöhten Meßwertanfalls treten dann Probleme der Datenverarbeitung stärker in Erscheinung, von denen drei Teilaspekte gesondert zu betrachten sind:

a) Zusätzliche Vorrichtungen zur sicheren Identifizierung der Analysenergebnisse werden exforderlich.

b) Das Berechnen der Ergebnisse soll schnell und unkompliziert im Labor erfolgen, um Plausibilitätskontrollen zu ermöglichen und eine schnelle Weitergabe dringend benötigter Ergebnisse zu jeder Tageszeit zu gewährleisten.

c) Die Ergebnisse sollen in einer Form anfallen, die u. U. die mechanische Übernahme in eine größere Datenverarbeitungsanlage exlaubt, z. B. in Form eines Lochstreifens.

Die vorliegende Arbeit beschreibt, wie die genannten. Probleme mit elektronischen Kleinrechnern (Olivetti Programma 101 und 203P) angegangen werden können. Beispiele von Rechenprogrammen zum Auswerten von Autoanalyzer-Meßwerten werden angegeben, da diesse Geräte zur Zeit in Deutschland weit verbreitet sind.

\section{Identifikation der Proben}

Die . Probe und die Laborleistungs-Anforderung mit Angaben über den Patienten und einer 6stelligen. Konttollnummer lassen sich mechanisch miteinander verbinden, so daß während der Aufarbeitung Verwechslungen nicht vorkommen sollten. Ein entsprechender Entwurf einer Anforderungskarte der Firma IBM für das System IBM 1080 erscheint allgemein anpassungsfähig und verwendbar (s. Abb. 1). Für den Technicon-Autoanalyzer wird neuerdings eine Vorrichtung angeboten, die die Kontrollnummer der Probe von der Kurzlochkarte abliest und auf das Registrierpapier aufdruckt. Damit stehen Meßwert und Kontrollnummer nebeneinander zur Weiterverarbeitung bereit. Es erscheint sinnvoll, den in Abbildung 1 gezeigten Vordruck für alle Laboranfor-

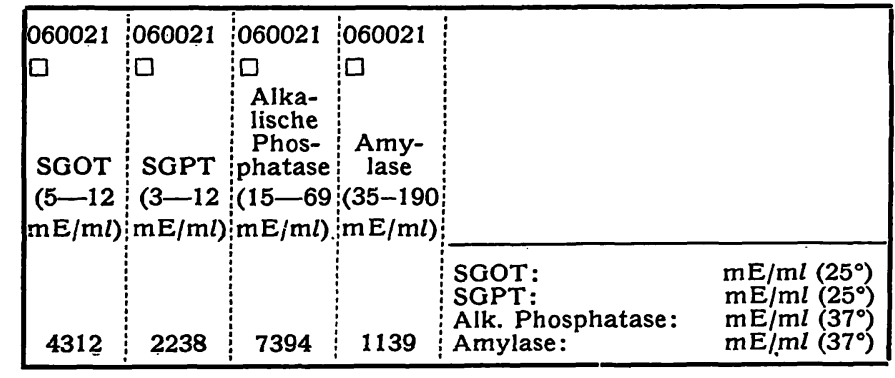

Kurzlochkarten Stammabschnitt

Abb. 1

Muster einer Labor-Anforderungskarte

In die Kurzlochkarten ist in der Praxis noch die 6 stellige PatientenNr. gestanzt

derungen zu verwenden. Der Übergang zu vollmechanischen Analysensystemen zu einem späteren Zeitpunkt verlangt dann keine Umstellungen.

Die für das Klinikum Berlin-Steglitz modifizierte Laboranforderung (Abb. 1) hat das Format einer IBM-Standardlochkarte $(8 \times 20 \mathrm{~cm})$. Die rechte Hälfte, der sogenannte Stammabschnitt, enthält im oberen Teil den Aufdruck einer Adremaplatte mit den Angaben über den Pătienten. Auf der unteren Hälfte werden die Ergebnisse eingetragen. Die linke Seite des Vordrucks enthält bis zu 4 abtrennbare Kurzlochkarten, in die die 6 stellige Aufnahme-Nr. des Patienten gestanzt ist und auf die diese Nummer in 


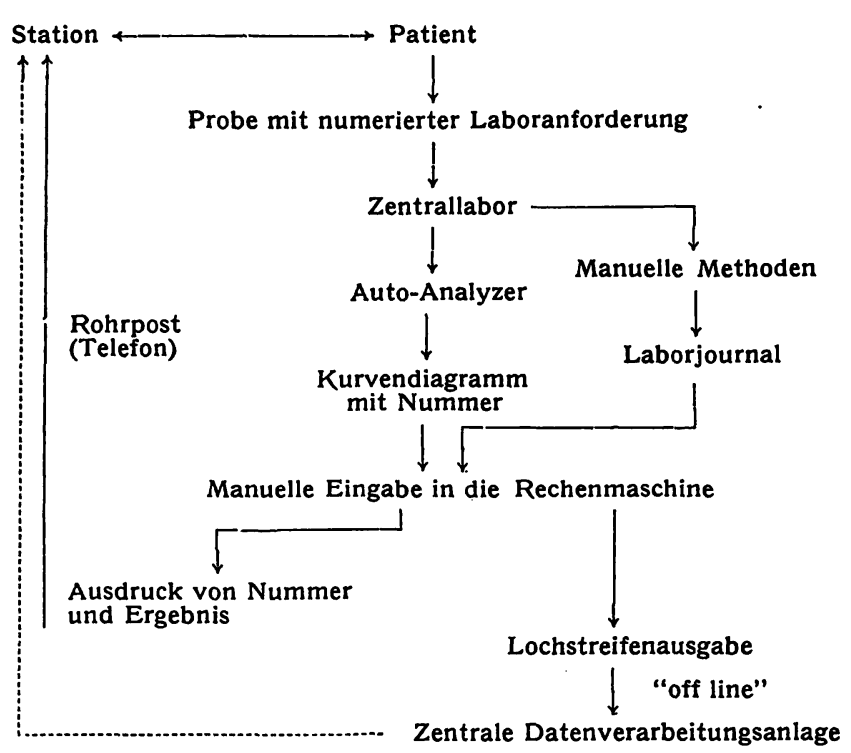

Abb. 2

Probenidentifikation und Analysenberechnung

Klartext gedruckt ist. Auf der Kurzlochkarte ist die gewünschte Analyse angekreuzt. Die Karten begleiten das Analysenmaterial durch das Labor und sichern die Identifizierung der Probe(s. Abb. 2). Durchschläge der Laboranforderung dienen als Leistungsbeleg für die Verwaltung und zu internen Kontrollzwecken.

\section{Mathematische Grundlagen}

Programmbeispiele für die Olivetti Programma 101

Ein großer Teil der Meßergebnisse des klinisch-chemischen Zentrallabors fällt bei photometrischen Endwertmessungen an. An Hand dieses Beispiels soll deshalb der Einsatz des Rechners beschrieben werden.

\section{Programm a}

Bei manuellen Methoden, die auf Absorptionsmessungen hinaus!aufen, wird man meist die Bedingungen so einrichten können, daß das LAMBERT-BEERsche Gesetz erfüllt ist, $d . h$. eine lineare Beziehung zwischen Meßwert (Extinktion) und abhängiger Größe (Konzentration) vorliegt. Ist der Extinktionskoeffizient eine aus Eichwerten jeweils neu zu ermittelnde Größe, kann man durch die Eichpunkte (E/c) eine Ausgleichsgerade nach der Methode der kleinsten Fehlerquadrate legen (1) und daraus für alle anfallenden Meßwerte $\mathrm{E}$ die Konzentrationen c berechnen. Das im Anhang beschriebene Programm a führt diese Rechnungen aus.
Das Programm a, das sich auch für manuelle Methoden verwenden läßt, ist speziell für Auto-Analyzer-Methoden geschrieben, bei derien das LAMBERT-BeERsche Gesetz erfüllt ist (Beispiele: Kreatinin im Serum, HarnstoffStickstoff im Serum, s. Tab. 1). Das Registrieren der Extinktion und nicht der Transmission ist hier am zweckmäßigsten. Muß man aus technischen Gründen lineares Registrierpapier verwenden, benötigt man ein etwas größeres Rechenprogramm, das aus Transmissionswerten die Ergebnisse berechnet und etwas längere Rechenzeiten erfordert. (Das Programm ist auf Wunsch beim Verfasser erhältlich).

\section{Programm $b$}

Bei nichtlinearen Eichkurven ist die Wahl des Registrierpapiers, d. h. der Koordinaten, noch von größerem Einfluß auf die erzielbare Genauigkeit. Fast alle üblichen Auto-Analyzer-Methoden sind Relativmethoden. In der Praxis interpoliert man meist linear zwischen empirischen Stützpunkten, deren Anzahl sich aus Gründen der Leistung nicht beliebig ethöhen läßt. Je weniger eine solche Eichkurve gekrümmt ist, desto besser ist die lineare Interpolation bei gleicher Anzahl der Stützpunkte. Abbildung 3 zeigt eine konstruierte empirische Eichkurve, die bei hohen Konzentrationen eine Abweichung vom LAMBERT-BEERschen Gesetz zeigt. Die Darstellung der Abbildung 3a entspricht der vielfach geübten manuellen Auswertung. Registriert man statt der Transmissionswerte jedoch Extinktionen, erhält man die viel weniger gekrümmte Eichkurve der Abbildung $3 \mathrm{~b}$ und somit genauere Ergebnisse. Es gibt nun unter anderen auch AutoAnalyzer-Methoden, bei denen die Aufhellung einer gefärbten Lösung gemessen wird (Beispiele: Blutglucose nach der Ferricyanid-Methode, proteingebundenes Jod). Hier bleibt die lineare Interpolation praktisch als einzige Auswertungsmethode übrig. Im speziellen Fall der Glucose-Bestimmung, für die das Programm b geschrieben ist, empfiehlt sich das Registrieren der Transmissionswerte (Abb. 4). Es werden 6 Stützpunkte vorgegeben. Zur Vereinfachung des Programms werden die Eichlösungen als ganzzahlige Vielfache eines Grundbetrages $\Delta \mathrm{C}$ angesetzt. $\Delta \mathrm{C}$ wird als Konstante vom Programm generiert. Die Auswahl der Eichpunkte (in diesem Fall: 0, 50, 100, 150, 200, $250 \mathrm{mg}$ Glucose $/ 100 \mathrm{~m} /$ ) ist damit für die jeweilige Methode festgelegt (Abb. 4). Durch Ändern einiger Befehle kann das Programm b anderen nichtlinearen Methoden leicht angepaßt werden. Geeignet sind

Tab. 1

Rechnerischer Ausgleich einer Eichgeraden $c=a \cdot E+b$ nach der Methodeder kleinsten Fehlerquadrate. Methode: Harnstoff-N mit dem Autoanalyzer 60 Proben/Std.

\begin{tabular}{|c|c|c|c|c|c|c|c|c|}
\hline $\mathrm{C}_{\text {Eichlsg. }}(\mathrm{mg} / 100 \mathrm{ml})$ & 0 & 10 & 20 & 30 & 40 & 50 & 100 & 200 \\
\hline Extinktion & 0,011 & 0,056 & 0,106 & 0,147 & 0,192 & 0,238 & 0,468 & 0,930 \\
\hline$\Delta \mathrm{C}(\mathrm{mg} / 100 \mathrm{ml})$ & $+0,1$ & $-0,1$ & $+0,8$ & $-0,3$ & $-0,5$ & $-0,4$ & $-0,6$ & $+0,2$ \\
\hline
\end{tabular}




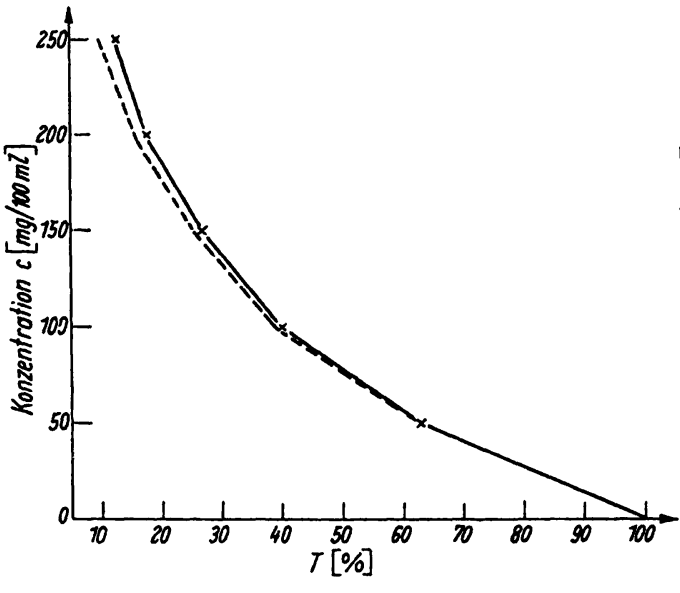

$a$

Abb. 3

Lineare Interpolation einer empirischen Eichkurve $x-x$. Zum Vergleich ist eine dem Lambeert-Beer'schen Gesetz folgende Eich kurve $(\stackrel{\longrightarrow}{\longrightarrow}$ mit eingezeichnet Darstellung von $(a)$ als Funktion der Transmission b) als Funktion der Extinktion

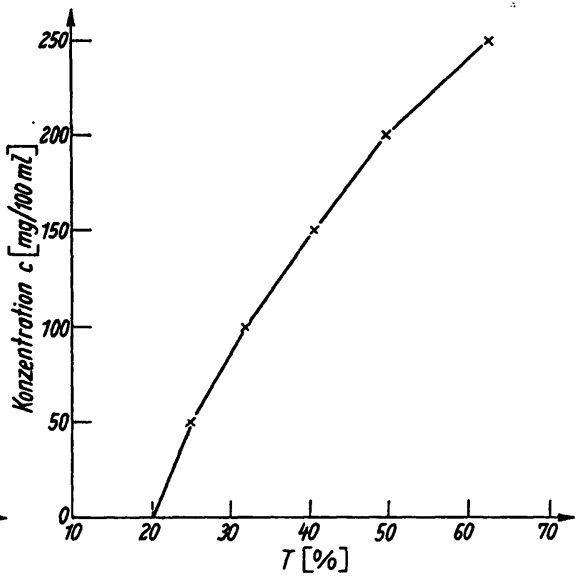

Abb. 4

Bestimmung von Glucose nach der Ferricyanid-Methode. (60 Proben/Std.) Eichkurve $\mathrm{c}=\mathrm{f}(\mathrm{T})$

Lineare Interpolation mit Rechenprogramm b besonders solche Methoden, die Eichkurven wie die in Abbildung 3b gezeigte liefern (Beispiel: Bilirubin im Serum mit dem Auto-Analyzer). Die Fehlerbreite der Rechenmethode läßt sich nicht allgemein angeben und entspricht der Fehlerbreite einer manuellen Auswertung. Durch geeignete Auswahl der Eichpunkte sollte der Normalbereich optimal approximiert werden. (Das Programm b wurde gemeinsam mit Herrn Dipl.Math. MarkwirTh für den etwas größeren Rechner 203P für maximal 10 Stützpunkte umgeschrieben. Dieses Programm ist bei der Deutschen Olivetti GmbH., Königstein/Taunus, erhältlich.)

\section{Praktische Bedienung:}

Der Ablauf der Bedienungsvorgänge ist aus den im Anhang dargestellten Programmen ersichtlich. Jedes Programm besteht im Prinzip aus 2 Teilen. Zuerst werden die Eichwerte eingegeben und so verarbeitet, $\mathrm{da} ß$ die im zweiten Teil benötigten Konstanten bereitstehen. Zum Berechnen der Proben wird dann die Patienten-Nr. eingetastet und zusammen mit der codierten Analysen-Nr., die das Programm generiert, ausgedruckt. Aus dem dann eingegebenen Meßwert wird das Endergebnis berechnet und - falls wünschenswert, abgerundet - ausgedruckt. Das Rechnen mit der Programma 101 erfordert bei Vorliegen fertiger Programme nur eine kurze Einweisung ähnlich wie bei in der Laborroutine üblichen Analysengeräten (medizinisch-technische Assistentinnen haben nach unșerer Erfahrung keine Schwierigkeit in der Bedienung). Für jede Analysenart liegt ein Programm auf einer Magnetkarte vor, von der es im Bedarfsfall auf den Rechner übertragen wird. Die Rechenzeiten der zur Zeit von uns angelegten 12 Programme liegen zwischen 3 und 15 Sekunden pro Analysenwert. Der Aufwand für Pflege und Wartung ist bei bisher 15 Monaten Betriebsdauer gering.
Datenweitergabe an eine zentrale Datenverarbeitungsanlage .

Nach Auskunft des Herstellers (Februar 1968) ist das größere Modell 203P im Herbst 1968 mit einer Lochstreifenausgabe erhältlich. Für den hier skizzierten Zweck reicht der kleinere und preiswertere Rechner Programma 101 jedoch aus. Ob für dieses Modell eine Lochstreifenausgabe erhältlich sein wird, steht nach Auskunft des Herstellers noch nicht fest. Auf den Lochstreifen sollten mindestens folgende Daten übertragen werden:

1. Die Patienten-Nummer (in unserem Fall 6stellig)

2. Die Analysenart, z. B. als 4 stellige $Z$ ahl codiert und

3. Das Ergebnis als 3- bis 4stellige Zahl.

Entsprechender Speicherplatz für einige zusätzliche Übertragungsbefehle ist in den mitgeteilten Programmen noch vorhanden.

\section{Diskussion}

Wie eine Umfrage in Berliner und westdeutschen Krankenhäusern ergab, steigt die Anzahl der anfallenden Analysenproben im klinisch-chemischen Zentrallabor'stetig an. Die Angaben schwankten zur Zeit zwischen 0,7 und 1,6 quantitativen chemischen Analysen pro Tag und Patient. Diese wachsenden Anforderungen verlangen nicht nur eine technisch sinnvolle und wirtschaftlich vertretbare Ausstattung mit sogenannten Analysenautomaten, sondern auch Eintichtungen, die eine schnelle Weitergabe von Befunden an den behandelnden Arzt erlauben. Nach unserer Auffassung ist der Einsatz eines Kleinrechners eine ebenso wichtige Rationalisierungsmaßnahme wie das Anschaffen von vollmechanischen Analysengeräten. Wir stellen an ein solches Gerät folgende Minimalforderungen: Es soll schreibend, extern program- 
mierbar sein und leicht zu bedienen, mindestens 10 numerische Daten speichern können und auf Wunsch zusätzlich eine Lochstreifenausgabe haben. Die hier beschriebene Lösung ist ein Kompromiß, der auch den wirtschaftlichen Gesichtspunkt mit berücksichtigt und deshalb auch für mittlere Krankenhäuser vertretbar erscheint. Die Flexibilität der Programme, die einfache Bedienung und der für alle Beteiligten unkomplizierte Arbeitsgang (s. Abb. 2), der zwischendurch Plausibilitätskontrollen erlaubt, sind gewichtige Vorteile eines laboreigenen Kleinrechners. Auch in Laboratorien, die mit größeren Digitalrechnern ausgestattet oder verbunden sind, hat nach unserer Auffassung der Kleinrechner eine Existenzberechtigung. Nach dem gegenwärtigen Stand der Technik macht es große Mühe, alle Meßgeräte eines Zentrallabors mit einem Digitalrechner im "on line“-Betrieb abzufragen. Der technische und finanzielle Aufwand ist dafür wesentlich höher. Infolge der Vielfalt aller vorhandenen Geräte werden auch beim Vorhandensein eines solchen Prozeßrechners immer noch Daten von Geräten anfallen, deren Meßergebnisse manuell in den Kleinrechner übertragen werden müssen. Der Kleinrechner überbrückt außerdem bequem die Ausfallund Reparaturzeiten des Großrechners.

\section{Anhang}

\section{Rechenprogramme für die Programma 101}

\section{Programm a}

Berechnen von Analysendaten aus einer Regressionsgeraden (z. B. Kreatinin mit dem Autoanalyzer).

\section{Beschreibung der Arbeit}

Für eine Serie von Meßwerten (E/c) existiert eine lineare Beziehung zwischen $c$ und $E$ :

$$
c=a E+b
$$

$a$ und $b$ sind von Serie zu Serie etwas wechselnde Größen, die durch Analyse von Eichlösungen ermittelt werden müssen. Durch die n Eichpunkte $\left(c_{E} / E_{E}\right)$ wird eine Ausgleichsgerade $\bar{c}$ nach der Methode der kleinsten Fehlerquadrate gelegt. Die Bestimmungsgleichungen lauten:

$$
\begin{aligned}
& \mathrm{b} \cdot \mathrm{n}+\mathrm{a} \cdot \Sigma \mathrm{E}_{\mathrm{E}}=\Sigma \mathrm{c}_{\mathrm{E}} \\
& \mathrm{b} \cdot \Sigma \mathrm{E}_{\mathrm{E}}+\mathrm{a} \cdot \Sigma \mathrm{E}_{\mathrm{E}}^{2}=\Sigma \mathrm{cE}_{\mathrm{E}} \cdot \mathrm{E}_{\mathrm{E}} .
\end{aligned}
$$

Die Auflösung nach der Cramerschen Regel ergibt:

$$
\begin{gathered}
\mathrm{D}=\mathrm{n} \cdot \Sigma \mathrm{E}_{\mathrm{E}}^{2}-\left(\Sigma \mathrm{E}_{\mathrm{E}}\right)^{2} \\
\mathrm{D}_{\mathrm{a}}=\mathrm{n} \cdot \Sigma \mathrm{c}_{\mathrm{E}} \cdot \mathrm{E}_{\mathrm{E}}-\Sigma \mathrm{E}_{\mathrm{E}} \cdot \Sigma \mathrm{c}_{\mathrm{E}} \\
\mathrm{D}_{\mathrm{b}}=\Sigma \mathrm{CE}_{\mathrm{E}} \cdot \Sigma \mathrm{E}_{\mathrm{E}}^{2}-\Sigma \mathrm{E}_{\mathrm{E}} \cdot \Sigma \mathrm{c}_{\mathrm{E}} \cdot \mathrm{E}_{\mathrm{E}} \\
\mathrm{a}=\frac{\mathrm{D}_{\mathrm{a}}}{\mathrm{D}} \quad \mathrm{b}=\frac{\mathrm{D}_{\mathrm{b}}}{\mathrm{D}}
\end{gathered}
$$

\section{Ablaufbeschreibung}

\section{Vorgang}

Stellung des Dezimalrades 4

1. Netzschalter einschalten.

2. L̈̈schtaste drücken.

3. Magnetkarte durchlaufen lassen

4. Taste ' 'Z' drücken.

5. Extinktion des Eichwertes $E_{E}$ eintasten

6. Taste ' $S$ ' drücken.

7. Konzentration des Eichwertes $C_{E}$ eintasten

8. Taste ' $S$ ' drǘcken.

9. Schritte $5-8$ mit allen Eichpunkten $\left(E_{E} / c_{E}\right)$ wiederholen.

10. Taste ' $Y$ ' drücken. 11. Es wurden $a$ und $b$ ausgedruckt mit
$A \diamond$ und $B \backslash$

2. Die Patienten-Nr. wird eingetastet.

13. Taste 'S' drücken.

14. Die Code-Nr. der Analyse wird ausgedruckt.

15. Den Extinktionswert 'E' der Probe eintasten.

16. Taste ' $S$ ' drücken.

17. Das Ergebnis $c$ wird, auf eine Nachkommastelle abgerundet, ausgedruckt.

18. Zum Berechnen weiterer Proben werden die Schritte $12-17$ wiederholt.

Programmbefehle $\left.^{1}\right)$ (76 Befehle)

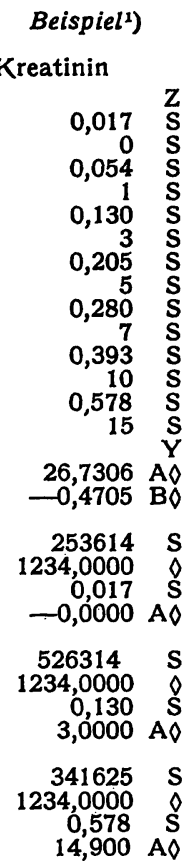

Die Befehle sind spaltenweise von oben nach unten zu lesen

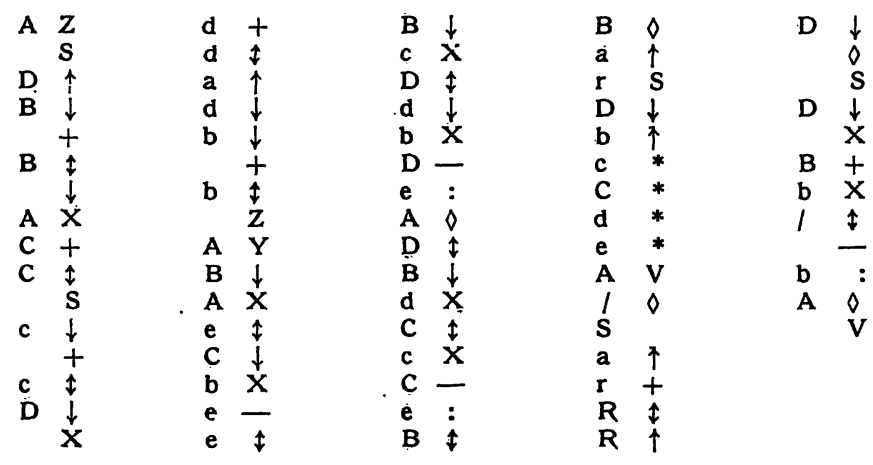

\section{Programm $b$}

Lineare Interpolation einer empirischen Eichkurve mit 6 Stütıpunkten, 1 Karte, $15+90$ Befehle.

\section{Beschreibung der Arbeit}

Es ist eine empirische Eichkurve mit 6 Stützpunkten gegeben (vgl. Abb. 4). Für gegebene Meßwerte I soll die abhängige Variable $\mathrm{c}$ durch lineare Interpolation berechnet werden. Durch ein System bedingter Sprünge wird das Intervall, in dem I liegt, festgestellt und $c$ nach folgenden Bestimmungsgleichungen berechnet:

Intervall:

1. $\mathrm{I}_{0}<\mathrm{I} \leqq \mathrm{I}_{1}$

2. $\mathrm{I}_{1}<\mathrm{I} \leqq \mathrm{I}_{2}$

3. $\mathrm{I}_{2}<\mathrm{I} \leqq \mathrm{I}_{3}$

4. $\mathrm{I}_{3}<\mathrm{I} \leqq \mathrm{I}_{4}$

5. $\mathrm{I}_{4}<\mathrm{I}$
Bestimmungsgleichung:

$$
\begin{aligned}
& c_{1 i}=\Delta c\left[\frac{I-I_{0}}{I_{1}-I_{0}}\right] \\
& c_{2 i}=\Delta c\left[1+\frac{I-I_{1}}{I_{2}-I_{1}}\right] \\
& c_{3 i}=\Delta c\left[2+\frac{I-I_{2}}{I_{3}-I_{2}}\right] \\
& c_{41}=\Delta c\left[3+\frac{I-I_{3}}{I_{4}-I_{3}}\right] \\
& c_{51}=\Delta c\left[4+\frac{I-I_{4}}{I_{5}-I_{4}}\right]
\end{aligned}
$$

$\Delta c=50$ (Befehle 88, 89).

1) Die Symbole sind im Bedienungshandbuch zur Programma 101 ausführlich erklärt. 
Ablaufbeschreibung

\section{Vorgang}

Stellung des Dezimalrades: 3

1. Programmkarte b1 durchlaufen lassen.

2. Taste ' $Z$ ' drücken.

3. Eichwert $I_{0}$ eintasten.

4. Taste 'S' drücken.

Schritte 3 u. 4 mit $I_{1}, I_{2}, I_{2}, I_{4}, I_{6}$ wiederholen. Anschließend Programm b2 durchführen.

Nicht die Löschtaste drücken!

6. Programmkarte b2 durchlaufen lassen.

7. Taste ' $W$ ' drücken.

8. Patienten-Nr. eintasten.

9. Taste ' $S$ ' drücken.

10. Die Analysen-Nummer wird mit $\diamond$ ausgedruckt.

11. Meßwert ' $I$ ' eintasten

12. Taste' ' $S^{\prime}$ drücken.

13. Das Ergebnis wird mit A๑ ausgedruckt.

14. Schritte 8-13 mit weiteren Meßwerten I wiederholen.

\title{
Programmbefehle
}

Die Befehle sind spaltenweise von oben nach unten zu lesen Register $M, A, R$ Operanden

Register B/, B, C/, C, D/, D, R Speicherungen

Register E/, E, F/, F Befehle

A $\mathbf{z}$

$\mathbf{s}$

b $\uparrow$

A W

R $\mathrm{S}$

A $\mathbf{V}$

10

M S

a $\uparrow$

$r+$

$\begin{array}{ll}\mathbf{R} & \mathbf{1} \\ \mathbf{R} & \uparrow\end{array}$

R

M

M S

$M \downarrow$

B

l v

B +

b 1234,000 (

40,5

$150,000 \mathrm{~A} \bigcirc$

$365214 \mathrm{~S}$

1234,000

77,700 AO

$415263 \mathrm{~S}$

1234,000 i

200,000 AO

b1

$\begin{array}{clllll}\text { c } & \uparrow & \text { S } & \text { D } & \uparrow \\ & S & \text { d } & \uparrow & \text { R } & S\end{array}$

b2

B $\downarrow$

$M-$

b

M :

M Z

a V

B +

i $\overline{\mathrm{w}}$

c +

B -

B

c $\downarrow$

$M$

B

$M$ :

$s$
$\uparrow$
$s$
$\downarrow$
$t$
$\vdots$
$v$
+
+
$\downarrow$
$\downarrow$
$\downarrow$
$\vdots$

a $\uparrow \quad M$

$M+$ a $Y$

M Z

a W

c + d -

c

Y Y

c +

c $\uparrow$

C $\downarrow$

M -

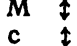

M :

a $\uparrow$

s

$\mathrm{S}$
$\mathrm{Z}$

d $\downarrow$

$M$

D

$M-\downarrow$

d $\uparrow$

M

a $\uparrow$

d +

$M+$

A Z

a $\quad \uparrow$

$\mathrm{r} s$

D $\mathrm{X}$

$\begin{array}{ll}\mathrm{A} & \mathrm{v} \\ \mathrm{M} & \mathrm{V}\end{array}$

\section{Literatur}

1. Zurmühr, R., Praktische Mathematik 5. Auflage, S. 339, Springer Berlin (1965).

Dr. K. Borner

1 Berlin 45, Klingsorstr. 95a

Klinikum der Freien Universität

\section{Papierchromatographische Untersuchungen von zur Anfärbung von Elektropherogrammen benutzten Farbstoffen}

\author{
I. Mitteilung zur Metbodik der Serumelektrophorese \\ Von V. Busse \\ Aus dem Institut für Angewandte Pbysiologische Chemie und Klinische Chemie der Freien Universität Berlin \\ (Direktor: Prof. Dr. H.-J. Dulce) \\ (Eingegangen am 13. Mãrz 1968)
}

Herrn Prof. Dr. Dr. Ernst Scbütte zum 60. Geburtstag gewidmet

Nur Ponceau $S$ ist ein chromatographisch einheitlicher Farbstoff und zur Anfärbung von Elektrophoresestreifen geeignet. Amidoschwarz 10B, Azokarmin G und B, Neucoccin, Bromphenolblau und Nigrosin enthalten Begleitfarbstoffe und erfüllen die an diese Methode gestellten Forderungen nicht.

Die Farbstofftrennungen wurden in 30 verschiedenen Fließmittelsystemen durchgeführt. Es wurden 3 für die Trennung besonders geeignete Systeme angegeben. Die damit erhaltenen Farbstoffkomponenten werden durch $R_{\mathrm{F}}$-Werte und Absorptionsmaxima charakterisiert.

Ponceau $S$ is the only chromatographically pure dye, suitable for staining electrophoresis strips. Amido black 10B, azocarmine $G$ and $B$, neucoccin, bromophenol blue and nigrosin contain impurities and do not satisfy the requirements of the method.

The dyes were resolved in $\mathbf{3 0}$ different solvent systems. Three systems are reported, which are especially suitable. The dye components thus obtained were characterised by $R_{F}$ and absorption maxima.

Zum Sịchtbarmachen und zum Auswerten von elektrophoretisch aufgetrennten Serumproteinen werden Farbstoffe verwendet. Sollen die durch Elution oder automatische Extinktionsschreibung ermittelten Farbstoffkonzentrationen quantitativ reproduzierbar sein, müssen diese Farbstoffe bestimmte Forderungen erfüllen:
1. Schnelle und durchdringende Färbung aller Proteine

2. Schnelle und gründliche Auswaschbarkeit des nicht an Protein gebundenen Farbstoffs

3. Lineare stöchiometrische Beziehung zwischen Farbstofflbindung und Eiweißgehalt 